\title{
Insulin Pump Therapy Issues Among Adults with Type 1 Diabetes Mellitus in Saudi Arabia: A Retrospective Study
}

\author{
Yousef Al-Saleh (1) · Fajr Al Motairi · Esra Hassan · Abdullah Al Sohaim • \\ Ibtisam Al Anazi · Ashwag Al Masoud · Rasha Al Wohabe • \\ Sultana Al Anazi · Awad Al Shahrani · Nasser M. Al-Daghri
}

Received: September 2, 2020 / Accepted: October 6, 2020 / Published online: October 17, 2020

(C) The Author(s) 2020

\section{ABSTRACT}

Introduction: Type 1 diabetes mellitus (T1DM) is on the rise in Saudi Arabia. Management of T1DM is crucial in curbing the economic burden of this disease. Studies on insulin pump issues are scarce in the region. The present study aims to fill this gap.

Methods: In this single-centre, retrospective study done in King Abdulaziz Medical City (KAMC) from March 2018 to March 2019, a

Y. Al-Saleh $(\bowtie) \cdot$ A. Al Shahrani

College of Medicine, King Saud Bin Abdulaziz

University for Health Sciences, Riyadh 22490, Saudi

Arabia

e-mail: yousef.alaslawi@gmail.com

Y. Al-Saleh · E. Hassan · A. Al Sohaim .

A. Al Shahrani

King Abdullah International Medical Research

Center, Riyadh 11481, Saudi Arabia

Y. Al-Saleh · E. Hassan · A. Al Sohaim .

A. Al Shahrani

Department of Medicine, King Abdulaziz Medical

City, Ministry of National Guard-Health Affairs,

RiyadhRiyadh 14611, Saudi Arabia

Y. Al-Saleh · N. M. Al-Daghri

Chair for Biomarkers of Chronic Diseases, College of Science, King Saud University, Riyadh 11451, Saudi Arabia

F. Al Motairi · I. Al Anazi - A. Al Masoud .

R. Al Wohabe · S. Al Anazi

Department of Nursing, King Abdullah Specialized

Children Hospital, Riyadh, Saudi Arabia total of 118 known Saudi T1DM adults (34 males and 84 females) were included. Data on demographics, glycated haemoglobin (HbA1c) and CSII use were collected.

Results: The most common problem encountered by the patients on CSII was breaking down of the pump (30.0\%), relocation of the cannula or tubing (22\%) and air bubbles affecting delivery (16.1\%). Eighty-one subjects (68.6\%) claimed to have been admitted for DM management while on CSII. The use of CSII led to a significant reduction in HbA1c in all subjects $(p<0.001)$, but levels remain suboptimal. Only 53 subjects $(44.9 \%)$ reported no significant problems in their CSII experience.

Conclusion: There is a high prevalence of T1DM adult patients experiencing device malfunctions and other issues while on CSII therapy. This may account for suboptimal improvement in the glycaemic control among T1DM Saudi patients. Issues on adherence and device malfunctions should be investigated further.

Keywords: HbA1c; Insulin pump; Saudi; Type 1 diabetes 


\section{Key Summary Points}

Why carry out this study?

Type 1 diabetes mellitus is on the rise in Saudi Arabia.

Studies on insulin pump use and associated issues among T1DM Arab adults are scarce in the region.

What was learned from the study?

Insulin pump therapy among Arab adults with T1DM sub-optimally improves glycaemic control.

Device malfunctions is the most common issue and this may negatively influence adherence.

\section{DIGITAL FEATURES}

This article is published with digital features, including a summary slide, to facilitate understanding of the article. To view digital features for this article go to https://doi.org/10.6084/ m9.figshare.13049705.

\section{INTRODUCTION}

Type 1 diabetes mellitus (T1DM) is an autoimmune disease previously known as insulin-dependent or juvenile-onset type diabetes [1], characterized by absolute insulin deficiency secondary to lack or total destruction of pancreatic $\beta$-cells. It is the most common type of diabetes among children. According to the International Diabetes Federation (IDF) Diabetes Atlas (9th edition), more than a million children worldwide have T1DM as of 2019 [2]. Glycaemic targets in patients with T1DM can be achieved through continuous subcutaneous insulin infusion (CSII) or insulin pump therapy [3]. This management was introduced $>$ 40 years ago and is still widely used globally for T1DM patients with well-defined clinical problems who must be interested, keen and able to undergo this therapy [3]. A crucial advantage of CSII compared to MDIs (multiple daily injection), especially in children, is its superior ease and flexibility [4]. Programmable basal rates are delivered routinely and with unparalleled precision, while bolus doses can be used effortlessly to regulate insulin exposure around meals and activities and to correct hyperglycaemia [4]. Among its major disadvantages are risk for ketoacidosis, infection at the infusion site and relatively higher cost compared to other insulin treatments [5].

The Kingdom of Saudi Arabia (KSA) ranks ninth out of the top ten countries with the highest incidence of T1DM per year as per the Diabetes Atlas (9th edition) [2]. However, in contrast to that in the developed countries, the number of research studies on the prevalence, incidence and sociodemographic aspects of T1DM is distressingly low [6]. Studies on Saudi T1DM adults is even fewer compared to the paediatric counterparts. Most of these studies focus on glycaemic control during Ramadan, the fasting month of Muslims where people with diabetes are at higher risk for complications $[7,8]$. A recent study by Almogbel compared the impact of pump therapy among T1DM Saudi users and observed that those under insulin pump had better glycaemic control over multiple-dose insulin injections (MDI), but had modestly more hypoglycaemic episodes per week [9]. Despite meaningful findings for the Saudi T1DM population, the previous study was non-randomized case-control and acknowledged that it failed to address several significant confounders such as patients' satisfaction, including issues encountered in adopting such technologies. Other studies on the use of CSII in the region were mostly qualitative in design focusing on children [10] or T2DM patients [11]. In this retrospective study, we determine the common issues encountered by Saudi T1DM adult patients on CSII therapy, their experiences and level of glycaemic control after CSII commencement. The present study is arguably the first in the region to address insulin pump issues among Arab T1DM adult patients. 


\section{METHODS}

This is a retrospective chart review conducted at the Adult Diabetes and Endocrinology Center, King Abdullah Specialized Children Hospital, King Abdulaziz Medical City (KAMC) in Riyadh, Saudi Arabia. Saudi adults with known T1DM aged 15-45 years old and who are on CSII therapy were included in the chart review. Exclusion criteria included T1DM patients who are $<$ years old, have severe diabetes complications, have learning disabilities and are not CSII therapy. Other types of DM as well as nonSaudis were also excluded. The study was performed in accordance with the Helsinki Declaration of 1964 and its later amendment. Ethical approval was obtained from the Institutional Review Board of KAMC, Riyadh, KSA (approval no. RC18/100/R). The Institutional Review Board of KAMC, Riyadh, also waived the need for informed consent.

At KAMC, CSII therapy is implemented following a training programme for patients and their parents/relatives. This training programme is led by the endocrinologist consultant and assisted by a multidisciplinary team for different settings. Throughout its use and after a period of maintenance on CSII, patients are followed up routinely, and for each visit, HbA1c is measured, which serves as guidance prior to needed insulin adjustments.

\section{Data Collection}

Demographic and clinical information was retrieved in charts of patients included. These included personal data (age and sex), age at diagnosis, duration of CSII, reasons for choosing CSII therapy, benefits of CSII therapy, problems encountered, hospital admissions, reasons for stopping CSII therapy, frequency of hypoglycaemic or hyperglycaemic episodes and glycated haemoglobin (HbA1c) levels at baseline and last follow-up. The type and model of insulin pump were also noted.

\section{Data Analysis}

Data were analysed using SPSS version 16.5 (SPSS Inc., Chicago, IL, USA). Continuous data were presented as mean \pm standard deviation for normally distributed variables and median (inter-quartile range) for non-normally distributed variables. Categorical data were presented as frequencies (\%). Independent $t$ test was done to compare parameters according to sex and paired $t$ test was done to compare baseline and follow-up HbA1c levels. Figures were plotted in MS Excel. Significance was set at $p<0.05$.

Table 1 General characteristics of T1DM subjects

\begin{tabular}{|c|c|c|c|c|}
\hline Parameter & All & Males & Females & $p$ value \\
\hline$N$ & 118 & 34 & 84 & \\
\hline Age (years) & $25.6 \pm 8.0$ & $22.8 \pm 8.3$ & $27.0 \pm 7.7$ & 0.01 \\
\hline Age at diagnosis (years) ${ }^{a}$ & $11(8-15)$ & $10.5 \pm 5.1$ & $12.5 \pm 7.2$ & 0.09 \\
\hline Age at first break (years) & $25.2 \pm 6.8$ & $20.8 \pm 5.1$ & $25.9 \pm 6.9$ & 0.12 \\
\hline Years on $\mathrm{CSII}^{\mathrm{a}}$ & $5(3-9)$ & $6.4 \pm 3.6$ & $5.4 \pm 3.3$ & 0.18 \\
\hline $\mathrm{HbAlc}$ at baseline & $9.0 \pm 2.2$ & $9.3 \pm 2.5$ & $8.9 \pm 2.1$ & 0.35 \\
\hline HbAlc at last follow-up & $8.1 \pm 1.8$ & $8.3 \pm 2.3$ & $8.0 \pm 1.5$ & 0.44 \\
\hline HbAlc paired $t$ test $p$ value & $<0.001$ & 0.008 & $<0.001$ & \\
\hline
\end{tabular}

${ }^{a}$ Non-normal data presented as median (inter-quartile range); $p$ value significant at $<0.05$ 


\section{RESULTS}

In the present retrospective, single-centre study, a total of 118 subjects [34 males aged $22.8 \pm 8.3$ years $(28.8 \%)$ and 84 females aged $27.0 \pm 7.7$ years $(71.2 \%) ; p=0.01]$ fulfilled the criteria for inclusion. Of the 118 patients included, 37 claimed to be admitted specifically for DM management while on pump (31.4\%). Of these 37, 19 (51.4\%) were confirmed in the system (not shown table). Table 1 shows the general characteristics of subjects. The mean age of subjects was $25.6 \pm 8.0$ years, with a median age of diagnosis at 11 years. The median average of CSII use was 5 years. Overall, while there was a significant improvement in HbA1c in all subjects, these changes were still clinically suboptimal. When stratified according to sex, females were significantly older than males $(p<0.01)$ and were slightly older at time of T1DM diagnosis $(p=0.09)$. There was no significant difference in age at first break, years using CSII as well as baseline and follow-up levels of HbA1c. Both males and females had significantly lower HbA1c levels at follow-up compared to baseline $(p=0.008$ and $<0.001$, respectively) (Table 1$)$. Between-group analysis adjusted for age showed no significant difference in HbA1c change.

\section{CSII}

At the time period of study, $13 \%(N=15)$ of subjects were not using insulin pump. Among the 103 users, 99\% were using Medtronic ${ }^{\circledR}$ (Medtronic Plc, Dublin, Ireland) while 2 (1\%) were on Omnipod ${ }^{\circledR}$ (Insulet Corp, Acton, MA, USA). Among Medtronic ${ }^{\circledR}$ users, most (61\%) were using MMT 722 followed by MMT 772 (34.7\%), MMT 640 (2.5\%) and MMT 754 (0.8\%) (not mentioned in tables).

\section{Common Problems, Reasons and Benefits Related to Using CSII}

Less than half of the patients $(N=53,44.9 \%)$ reported no significant problems in their CSII experience. The most common problem encountered by the patients on CSII was breaking down of the pump (30.0\%), relocation
Table 2 Common problems, reasons and benefits related to using CSII (all subjects)

\begin{tabular}{ll}
\hline Questions & $\begin{array}{l}\text { Percentage } \\
(\%)\end{array}$
\end{tabular}

Common problems when using CSII

Hypertrophy in injection site 3.4

Problems relocating cannula/tubing 22

Problems with air bubbles affecting insulin 16.1 delivery

Pump keeps breaking down 30.0

Consumables are too expensive 3.4

Inadequate support from pump company 0

Inadequate support from specialist 0.8 educator

I don't like wearing the pump

Gave little/no improvement in diabetes control

Difficult to use

No significant problems

Reasons for using CSII

I don't know

0

Recommended by doctor 58.5

Lifestyle reasons

Pregnancy

3.4

Improve awareness of sugar levels

Prevent long-term complications

27.1

For better diabetes control

Benefits of using CSII

Improved awareness of blood sugar

More convenient

Discreet when administering in public

Feels better

67.8

Relocating tubing is better than several

73.7 injections per day

Fits with lifestyle 74.6

Able to eat different foods 
Table 2 continued

\begin{tabular}{ll}
\hline Questions & $\begin{array}{l}\text { Percentage } \\
(\%)\end{array}$ \\
\hline Better control of diabetes & 74.6 \\
No benefit & 4.2 \\
\hline
\end{tabular}

of the cannula or tubing (22\%) and air bubbles affecting delivery (16.1\%). Around $15 \%$ of the subjects do not like wearing the pump (Table 2). Subjects had varying reasons for using the pump but most are aware that it is for diabetes control (59.3\%). More than half of all the participants are also using it because it was recommended by their physicians $(58.5 \%)$ and is compatible with their lifestyle $(52.5 \%)$. Less common reasons were to improve diabetes control $(27.1 \%)$ and to prevent long-term complications (22.9\%) (Table 2). Top benefits for using CSII according to subjects include better control of diabetes and fits with lifestyle (both at 74.6\%) and that relocation of tubing is more convenient than several injections per day (73.7\%). Most also thought it just feels better (67.8) and more convenient (55.9\%). Other listed benefits are included in Table 2.

\section{Hospitalizations, Breaks and Complications}

Eighty-one subjects $(68.6 \%)$ claimed to have been admitted for DM management while on CSII. Sixty-four subjects $(54.2 \%)$ had their last contact with their specialist within 3 months while $34.7 \%$ within 3-6 months. Most subjects (70.3\%) never had a break in CSII therapy and only $25.4 \%$ claimed to have one break while $4.2 \%$ had two breaks. Among the few subjects who stopped CSII, the most common reason was they do not like wearing pump (5.1\%) and found the pump was faulty (2.5\%) (not shown in tables). Severity of reported hypoglycaemia was mild in more than half of the subjects $(59.3 \%)$ while $37.3 \%$ reported moderate and $2.5 \%$ had severe hypoglycaemia. Frequency of hypoglycaemic attacks after starting on CSII and while on CSII is shown in Figs. 1 and 2 . More than one third (65.6\%) of the subjects reported having fewer hypoglycaemic attacks after CSII therapy while $13.6 \%$ reported more episodes (Fig. 1). More than half of the subjects reported having at least one episode in a week $(24.6 \%)$ or twice in a week (28.8\%) (Fig. 2).

\section{DISCUSSION}

The prevalence of DM in Saudi Arabia is continuously increasing and has been considered one of the most common chronic diseases contributing in the economic health burden in the Kingdom [12]. Achieving glycaemic control among Saudis with diabetes, regardless of type, is therefore a constant challenge not only for the patient but also for the entire medical community [13]. This retrospective study is arguably the first to address insulin pump issues among T1DM adult patients in the region.

The present study identified that the prevalence of device malfunction is high (30\%), followed by relocation of the cannula or tubing $(22 \%)$ and air bubbles affecting delivery $(16.1 \%)$. The prevalence of device malfunction is lower compared to other studies done elsewhere such as in New Zealand (38\%) [14] and France (68\%) [15]. Issues related to insulin pump malfunctions in general and Medtronic ${ }^{\circledR}$ in particular have been the most reported problems in the Food and Drug Authority (FDA) in the US [16]. Unfortunately, many companies will not reveal adverse events reported related to devices, but this type of information is useful for quality assurance and safety. As such, the need to address the safety concerns related to these devices and push the manufacturers to improve their designs aside from the usual warranty should be addressed. Furthermore, eligibility of users should be screened properly to prevent human errors and proper education, training and continuous technical provided to reduce the incidence of adverse events related to device malfunction [17].

As with CSII therapy, it is worth noting that only $53(44.9 \%)$ of the subjects reported no significant problems with CSII use. 


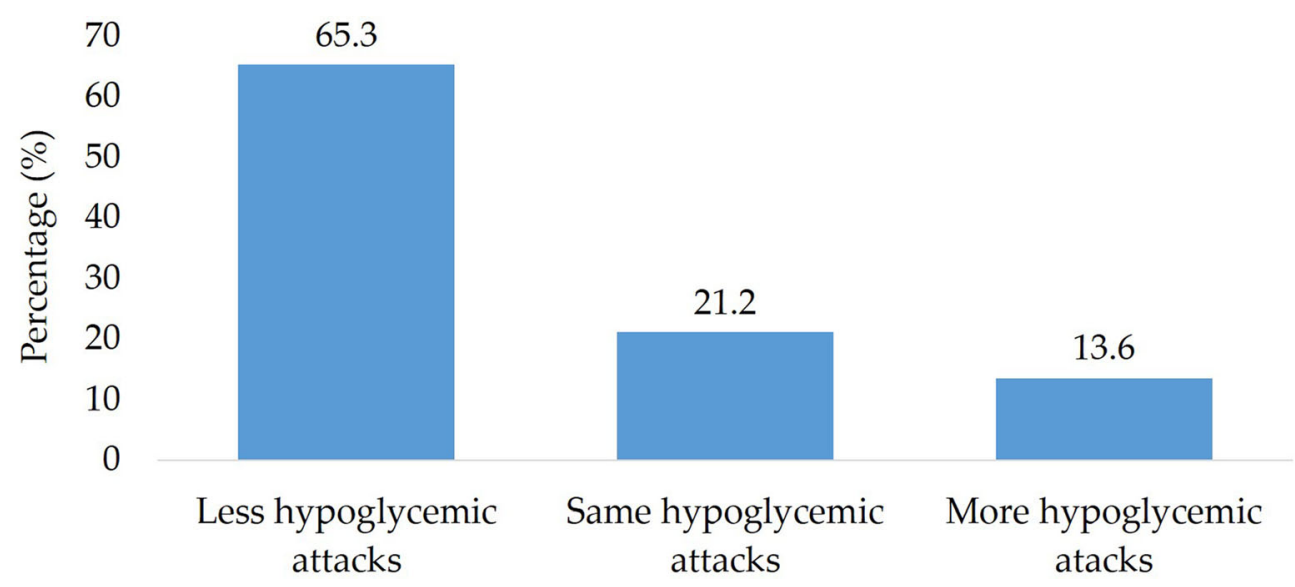

Fig. 1 Frequency of hypoglycaemic attacks after starting on CSII

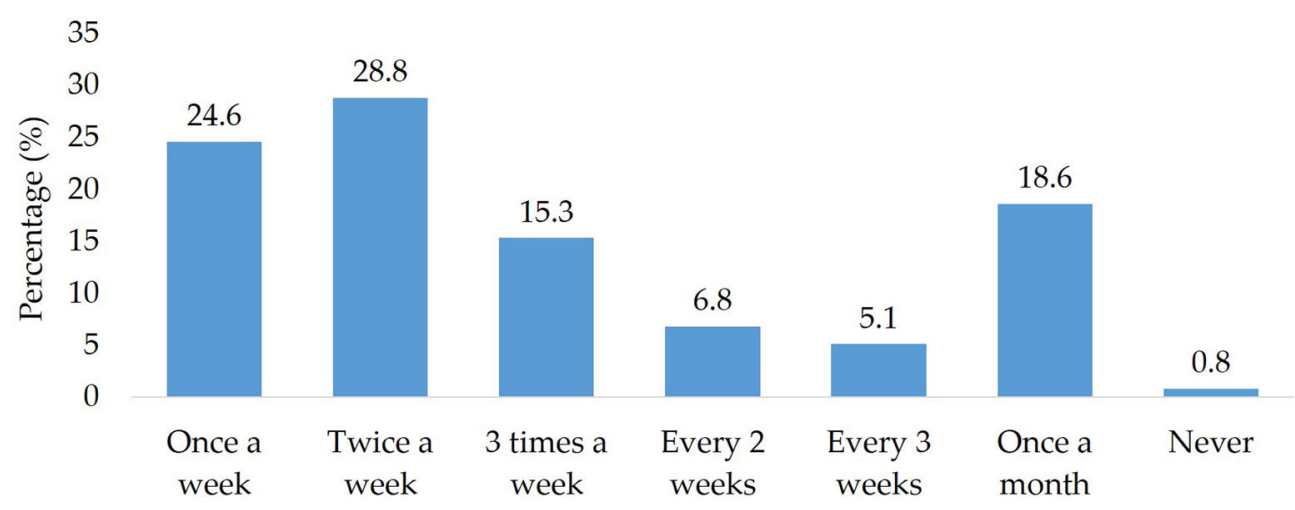

Fig. 2 Frequency of hypoglycaemic attacks while on CSII

Furthermore, 40 of the 118 subjects (33.8\%) reported having either no significant change in frequency of hypoglyacaemic attacks or more hypoglycaemic attacks after CSII therapy. CSII has been highlighted in several health economic studies as being more cost effective than MDI, at least in adults [18, 19], but not in children [20]. In the present study, the common issues encountered by the subjects regarding their medical devices should be reported to the Saudi Food and Drug Administration (SFDA) where approval of such devices takes place.

One of the major findings in the present study is that while most subjects are using CSII because of the convenience and compatibility with their lifestyle, the glycaemic control achieved by using CSII therapy remains suboptimal. This is true for both males and females.
The poor glycaemic control is also reflected by the high prevalence of hospital admissions in the cohort. A recent study by Alshareef and colleagues demonstrated a high correlation between hospital visits and glycaemic control among 530 adult Saudis with diabetes [21]. In their study, each unit of increase in HbA1c was attributed to a $43 \%$ increase in emergency department visits. They also highlighted that most participants were not followed up for emergence of complications 12 months prior to ER visits, a clear indication that diabetes care in KSA also has its own shortcomings [21].

Lastly, females outnumber males by almost $2: 1$, and this is consistent with the most recent epidemiological study conducted in KSA on T1DM [12] as well as the previous prospective study on T1DM and CSII use [22]. The sexual 
dimorphism in T1DM maybe more attributable to pathophysiological differences than the care provided, as significant variances have also been observed in other ethnic groups, with women having higher prevalence and being more likely to suffer from T1DM complications than men [23-25]. These gender differences in T1DM outcomes can also be attributed to differences in immune response and other external factors that underlie bias towards females especially with respect to autoimmune diseases [26]. Worth mentioning is that females were significantly older than males, confirming previous observations that autoimmune diseases in males are of young onset as opposed to acute onset in females [27].

The present study has several limitations. The design is retrospective and convenience sampling was used. Selection bias therefore cannot be avoided. The convenience sampling used however is homogeneous, as opposed to conventional convenience sampling, and the findings are more generalizable [28], albeit for a narrower population (Saudi T1DM adults). The findings are also limited to issues involving CSII therapy, since comparisons with other insulin delivery systems such as MDI have already been studied [9]. The present study nevertheless has merits, since it fills the gaps of previous observations that did not address insulin pump device issues in the assessment of its efficacy in achieving glycaemic control. Furthermore, the study adds value not only because of its comparatively larger sample size compared to previous local studies, but it is also the first to shed light on the issues and experiences of Saudi T1DM adult patients related to the use of CSII. Further investigations on an even larger scale (e.g. multi-centre) should be done on whether different insulin pumps show variances in efficacy among Saudi T1DM patients.

\section{CONCLUSIONS}

In summary, the prevalence of device malfunction is high among Arab adult T1DM users of CSII and this can partly explain the suboptimal improvement in their glycaemic control. Education and training need to be reemphasized among users for better outcome of insulin pumps. Issues on adherence and device malfunctions should be investigated further.

\section{ACKNOWLEDGEMENTS}

The authors thank the Chair for Biomarkers of Chronic Diseases, Deanship of Scientific Research at King Saud University, for the support.

Funding. Funding was provided by King Abdullah International Medical Research Centre (KAIMRC), Riyadh, Saudi Arabia. The Rapid Service Fee was funded by the authors.

Authorship. All named authors meet the International Committee of Medical Journal Editors (ICMJE) criteria for authorship for this article, take responsibility for the integrity of the work as a whole, and have given their approval for this version to be published.

Disclosures. Yousef Al-Saleh, Fajr Al Motairi, Esra Hassan, Abdullah Al Sohaim, Ibtisam Al Anazi, Ashwag Al Masoud, Rasha Al Wohabe, Sultana Al Anazi, Awad Al Shahrani and Nasser Al-Daghri have nothing to disclose.

Compliance with Ethics Guidelines. The study was performed in accordance with the Helsinki Declaration of 1964 and its later amendment. Ethical approval was obtained from the Institutional Review Board of KAMC, Riyadh, KSA (approval no. RC18/100/R). The Institutional Review Board of KAMC, Riyadh, also waived the need for informed consent.

Data Availability. All data generated or analysed in this study are included in the article.

Open Access. This article is licensed under a Creative Commons Attribution-NonCommercial 4.0 International License, which permits any non-commercial use, sharing, adaptation, distribution and reproduction in any medium or format, as long as you give appropriate credit to the original author(s) and the source, provide 
a link to the Creative Commons licence, and indicate if changes were made. The images or other third party material in this article are included in the article's Creative Commons licence, unless indicated otherwise in a credit line to the material. If material is not included in the article's Creative Commons licence and your intended use is not permitted by statutory regulation or exceeds the permitted use, you will need to obtain permission directly from the copyright holder. To view a copy of this licence, visit http://creativecommons.org/licenses/by$\mathrm{nc} / 4.0 /$.

\section{REFERENCES}

1. Diabetes Fact Sheet. World Health Organization 2019. https://www.who.int/news-room/fact-sheets/ detail/diabetes [Accessed July 23, 2020].

2. IDF Diabetes Atlas 9th edition 2019. https:// diabetesatlas.org [Accessed April 23, 2020].

3. Pickup J, Keen H. Continuous subcutaneous insulin infusion at 25 years: evidence base for the expanding use of insulin pump therapy in type 1 diabetes. Diabetes Care. 2002;25:593-8.

4. Campbell F. Review: The pros and cons of continuous subcutaneous insulin infusion (CSII) therapy in the paediatric population and practical considerations when choosing and initiating CSII in children. Br J Diabetes Vasc Dis. 2008;8:S6-S10.

5. Lenhard MJ, Reeves GD. Continuous subcutaneous insulin infusion: a comprehensive review of insulin pump therapy. Arch Intern Med. 2001;161(19): 2293-300.

6. Robert AA, Al-Dawish A, Mujammami M, et al. Type 1 diabetes mellitus in Saudi Arabia: a soaring epidemic. Int J Pediatr 2018:9408370.

7. Alfadhil EM. Higher rate of hyperglycemia than hypoglycaemia during Ramadan fasting in patients with uncontrolled type 1 diabetes: insight from continuous glucose monitoring system. Saudi Pharm J. 2018;26:965-9.

8. Alamoudi R, Alsubaiee M, Alqarni A, et al. Attitudes and habits of patients with type 1 diabetes during fasting Ramadan. J Clin Transl Endocrinol. 2018;14: $1-4$.
9. Almogbel E. Impact of insulin pump therapy on glycemic control among adult Saudi type-1 diabetic patients. An interview-based case-control study. J Family Med Prim 2020; Care 9: 1013-1019.

10. Bin-Abbas BS, Sakati NA, Al-Ashwal AA. Glycemic control and treatment satisfaction in Saudi diabetic children on insulin pump therapy. Ann Saudi Med. 2006;26:405.

11. Alsairafi ZK, Smith FJ, Taylor KMG, et al. A qualitative study exploring patients' experiences regarding insulin pump use. Saudi Pharm J. 2018;26:487-95.

12. Alotaibi A, Perry L, Gholizadeh L, Al-Ganmi A. Incidence and prevalence rates of diabetes mellitus in Saudi Arabia: an overview. J Epidemiol Glob Health. 2017;7:211-8.

13. Almaghaslah D, Abdelrhman AK, Al-Masdaf SK, et al. Factors contributing to non-adherence to insulin therapy among type 1 and type 2 diabetes mellitus patients in Asser region. Saudi Arabia Biomed Res. 2018;29(10):2090-5.

14. Ross P, Gray AR, Milburn J, et al. Insulin pumpassociated adverse events are common, but not associated with glycemic control, socio-economic status, or pump/infusion set type. Acta Diabetol. 2016;53:991-8.

15. Guenego A, Bouzillé G, Breitel S, et al. Insulin pump failures: has there been an improvement? Update of a prospective observational study. Diabetes Technol Ther. 2016;18:820-4.

16. Mohr $\mathrm{H}$, Weiss $\mathrm{M}$. Insulin pumps have most reported problems in FDA database. Medical XPRESS 2018; https://medicalxpress.com/news/ 2018-11-insulin-high-injuries.html [Accessed July $25,2020]$.

17. Heinemann L, Fleming GA, Petrie JR, et al. Insulin pump risks and benefits: a clinical appraisal of pump safety standards, adverse event reporting, and research needs: a joint statement of the European Association for the Study of Diabetes and the American Diabetes Association Diabetes Technology Working Group. Diabetes Care. 2015;38(4): 716-22.

18. Roze S, Smith-Palmer J, Delbaere A, et al. Cost effectiveness of continuous subcutaneous insulin infusion versus multiple daily injections in patients with poorly controlled type 2 diabetes in Finland. Diabetes Ther. 2019;10:563-74.

19. Doubova SV, Roze S, Ferreira-Hermosillo A, et al. Cost-effectiveness of the use of the continuous subcutaneous insulin infusion pump versus daily multiple injections in type 1 diabetes adult patients 
at the Mexican Institute of Social Security. Cost Eff Resour Alloc. 2019;17:19.

20. Blair JC, McKay A, Ridyard C, et al. Continuous subcutaneous insulin infusion versus multiple daily injection regimens in children and young people at diagnosis of type 1 diabetes: pragmatic randomised controlled trial and economic evaluation. BMJ. 2019;365:11226.

21. Alshareef SM, Aldayel AY, AlKhathlan MA, et al. Diabetic patients in Saudi Arabia: The evaluation of glycemic control measures based on emergency department utilization and the percentages of adherence to the recommended follow-ups for microvascular complications. Saudi Med J. 2019;40: 271-6.

22. Al Hayek AA, Robert AA, Al Dawish MA, et al. Efficacy of insulin pump therapy on diabetes treatment satisfaction and glycemic control among patients with type 1 diabetes mellitus in Saudi Arabia: a Prospective Study. Diabetes Ther. 2015;6:227-36.

23. Manicardi V, Russo G, Napoli A, et al. Gender-disparities in adults with type 1 diabetes: more than a quality of care issue. A cross-sectional observational study from AMD Annals Initiative. PLoS One 2016;11(10): e0162960.

24. Miller RG, Costacou T. Glucose management and the sex difference in excess cardiovascular disease risk in long-duration type 1 diabetes. Curr Diab Rep. 2019;19:139.

25. Luk AOY, Ke C, Lau ESH, et al. Secular trends in incidence of type 1 and type 2 diabetes in Hong Kong: a retrospective cohort study. PLoS Med. 2020;17:e1003052.

26. Ngo ST, Steyn FJ, McCombe PA. Gender differences in autoimmune disease. Front Neuroendocrinol. 2014;35:347-69.

27. Fairweather D, Frisancho-Kiss S, Rose NR. Sex differences in autoimmune disease from a pathological perspective. Am J Pathol. 2008;173:600-9.

28. Jager J, Putnick DL, Bornstein MH II. More than just convenient: the scientific merits of homogenous convenience samples. Monogr Soc Res Child Dev. 2017;82:13-30. 